\title{
A NEW BRANCH OF ENUMERATIVE GRAPH THEORY
}

BY W. T. TUTTE

Communicated by Walter Rudin, April 6, 1962

In a recent survey [1] F. Harary pointed out that the problem of enumerating planar graphs was important but still untouched. I am happy to be able to announce some results in this hitherto neglected field.

The results concern rooted maps, that is planar maps in which one edge is selected as the root, a positive sense of description is assigned to it and its two sides are distinguished as right and left. Thus a completely unsymmetrical map of $n$ edges gives rise to just $4 n$ rooted maps.

The number of combinatorially distinct rooted maps of $n \geqq 1$ edges is

$$
\frac{2(2 n) ! 3^{n}}{n !(n+2) !}
$$

Those of 1 and 2 edges are shown in Figure 1. In each diagram the arrow shows the directed root, and "right" and "left" have their usual meaning with respect to this arrow.

The number of nonseparable rooted maps of $n \geqq 1$ edges is

$$
\frac{2(3 n-3) !}{n !(2 n-1) !}
$$

There are 2 with 3 edges and 6 with 4 . These 8 rooted maps are illustrated in Figure 2. The figure shows unrooted maps, and each attached number shows in how many distinct ways the corresponding edge can be rooted.

The term " $c$-net" is sometimes used for a 3-connected map, circuits of 1 or 2 edges being excluded. The number $c_{n}$ of rooted $c$-nets with $n \geqq 4$ edges is given by

$$
c_{n+1}=2(-1)^{n+1}+R_{n},
$$

where

$$
R_{n}=\frac{(2 n+1) !}{8(n !)^{2}}\left\{\left(27 n^{2}+9 n-2\right) \int_{0}^{1} t^{n}(2 t-1)^{n} d t-(9 n-2)\right\} .
$$

$R_{n}=0$ when $n=0$, and other values of $R_{n}$ can be calculated from the recursion formula 


$$
\begin{aligned}
& d{ }^{2} \\
& d 1 b^{t} \\
& 088^{\circ}
\end{aligned}
$$




$$
S_{n} R_{n-1}+2 S_{n-1} R_{n}=\frac{2(2 n) !}{(n !)^{2}}, \quad S_{n}=27 n^{2}+9 n-2 .
$$

With the help of (4) $c_{n}$ has been computed as far as $c_{25}=1,932,856,590$.

The asymptotic formula for $c_{n}$ is

$$
c_{n} \sim \frac{2 n^{-5 / 2} 4^{n}}{243(\pi)^{1 / 2}} .
$$

If we assume that almost all unrooted $c$-nets are unsymmetrical we can obtain the corresponding formula for unrooted $c$-nets by dividing by $4 n$.

I know two methods of making planar enumerations. One is described in [2]. It deals with 3-connected rooted maps whose faces are all triangles. Such a map is called a simple triangulation if each circuit of 3 edges bounds a face. The number of simple triangulations with $n+3$ vertices is denoted by $\phi_{n, 0}$. The formula for $\phi_{n, 0}$ given in [2] can be transformed into one which resembles (3) and leads to the recursion formula

$$
\begin{aligned}
\phi_{n-1,0} T_{n}+4 \phi_{n, 0} T_{n-1} & =\frac{7(3 n) !}{n !(2 n+1) !}, \\
T_{n} & =8 n+5 .
\end{aligned}
$$

(Here $n \geqq 2$.) This result has made it possible to extend the table of values of $\phi_{n, 0}$ from $\phi_{8,0}=1173$ to $\phi_{21,0}=6,526,333,259,312$.

The second method starts with a region $R$ in the closed plane bounded by $s$ disjoint simple closed curves $J_{1}, J_{2}, \cdots, J_{s}$. On each curve $J_{i}$ an even number $2 n_{i}>0$ of points are specified as distinguishable "vertices." Let the number of combinatorially distinct ways in which $R$ can be dissected into simply connected domains by arcs in $R$ joining the vertices in pairs be denoted by $\gamma\left(n_{1}, n_{2}, \cdots, n_{s}\right)$. (Just one arc is to terminate at each vertex.) It is not difficult to find a recursion formula for $\gamma$, and it is easy to convince oneself by studying special cases that

$$
\gamma=\frac{(n-1) !}{(n-s+2) !} \prod_{i=1}^{8} \frac{\left(2 n_{i}\right) !}{n_{i} !\left(n_{i}-1\right) !}
$$

where $n=n_{1}+n_{2}+\cdots+n_{s}$. I found it possible, but not easy, to prove (7) from the recursion formula. The proof made use of the combinatorial identity 

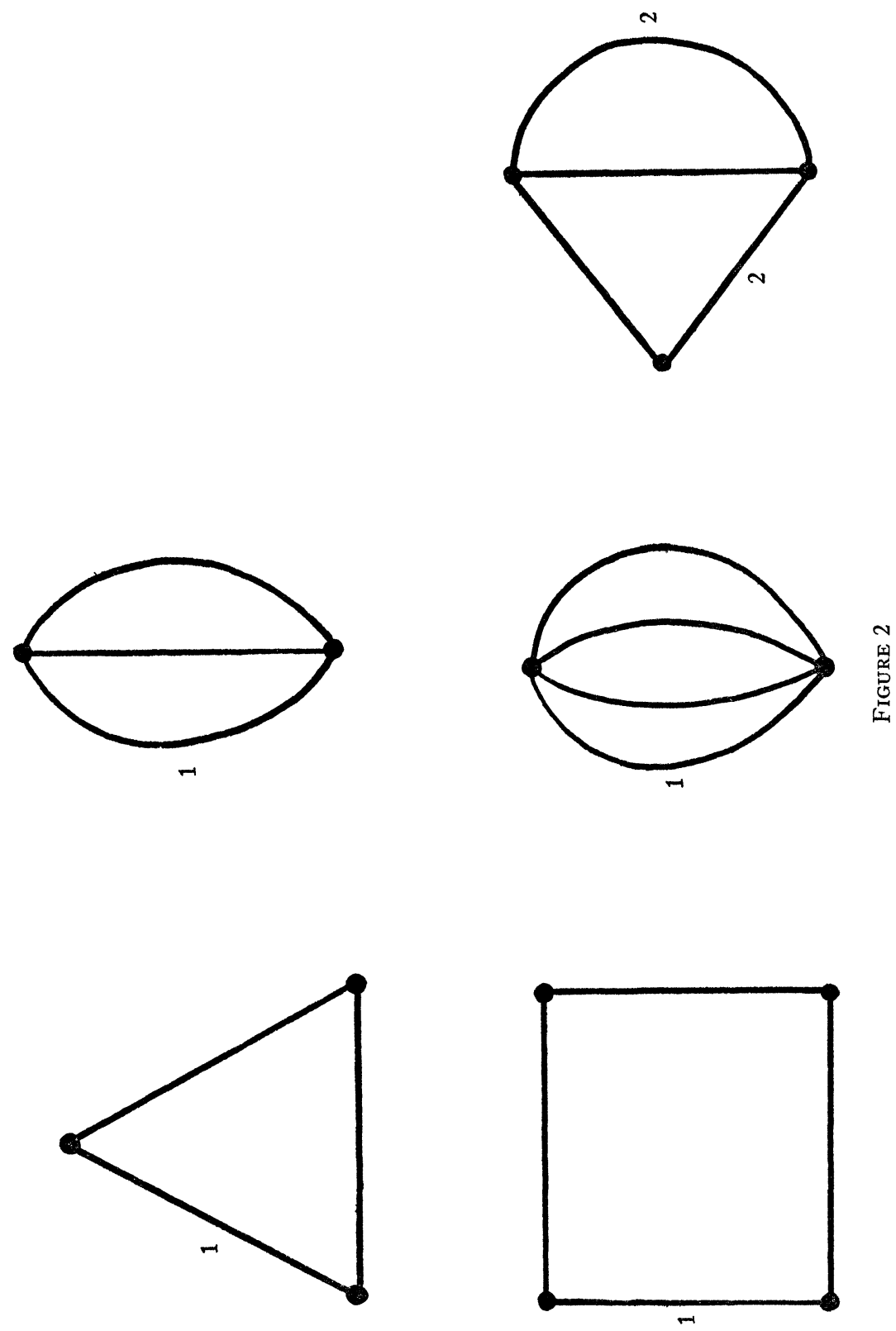


$$
\begin{aligned}
& \sum_{P} D^{\alpha(P)-k}\{\lambda\langle P\rangle\} \cdot D^{\alpha(\bar{P})-l}\{\mu\langle\bar{P}\rangle\} \\
& =\sum_{\alpha(P)<k} \sum_{i=0}^{k-1-\alpha(P)}(-1)^{i}\left(\begin{array}{c}
\alpha(\bar{P})-l \\
i
\end{array}\right) D^{\alpha(\bar{P})-l-i}\left\{D^{-k+\alpha(P)+i}\{\lambda\langle P\rangle\} \cdot \mu\langle\bar{P}\rangle\right\} \\
& +\sum_{\alpha(\bar{P})<l} \sum_{i=0}^{l-1-\alpha(\bar{P})}(-1)^{i}\left(\begin{array}{c}
\alpha(P)-k \\
i
\end{array}\right) D^{\alpha(P)-k-i}\left\{\lambda\langle P\rangle \cdot D^{-l+\alpha(\bar{P})+i}\{\mu\langle\bar{P}\rangle\}\right\} .
\end{aligned}
$$

Here $\lambda, \mu, f_{1}, f_{2}, \cdots, f_{s}$ are arbitrary functions of $x$, sufficiently often differentiable, $P$ is an arbitrary subset of $\left\{f_{1}, f_{2}, \cdots, f_{8}\right\}$, and $\bar{P}$ is its complementary subset. $\langle P\rangle$ is the product of the members of $P$. $D$ denotes differentiation with respect to $x$, and it is supposed that $D^{-1} f$ is defined as a particular integral for each relevant function $f$. $k$ and $l$ are fixed positive integers satisfying $s \geqq k+l-1 . \alpha(P)$ is the number of members of $P$.

I would be glad to learn more about this identity, which is new to me.

By contracting each curve $J_{i}$ to a single point we obtain from (7) a formula for the number of labelled Eulerian maps with a fixed consistent orientation of the faces, and with vertices of specified valencies. (We divide by $\prod_{i}\left(2 n_{i}\right)$.)

The case $n_{1}=n_{2}=\cdots=n_{s}=2$ corresponds to the duals of certain triangular subdivisions of the planar maps. It leads to formula (1). Formulae (2) and (3) can be derived from (1) by appropriate operations on generating functions.

The number $q_{n}$ of rooted bicubic maps, that is trivalent and bipartite maps, of $2 n$ vertices can be deduced from (7). It is

$$
\frac{6(2 n+1) ! 2^{n}}{n !(n+3) !}
$$

The rooted 3-connected bicubic maps of $2 n$ vertices are enumerated by a function $G(x)$ satisfying the functional equation

$$
F(x)=G\left(x\{1+F(x)\}^{3}\right),
$$

where $F(x)=q_{1} x+q_{2} x^{2}+q_{3} x^{3}+\cdots$.

\section{REFERENCES}

1. F. Harary, Unsolved problems in the enumeration of graphs, Publications Math. Inst. Hungar. Acad. Sci. 5 (1960), 63-95.

2. W. T. Tutte, A census of planar triangulations, Canad. J. Math. 14 (1962), 21-38.

UNIVERSITY OF TORONTO 